Research Article

\title{
Synthesis of 4,7,9-Trihydroxy[1]benzofuro[3,2-d] pyrimidine-6-carboxamide: Evaluation of Cytotoxicity and Inhibition of Protein Kinase C (CaPkc1)
}

\author{
Dao Viet Hung $\mathbb{D}^{1}$ and Tran Khac Vu $\mathbb{D}^{2}$ \\ ${ }^{1}$ Phu Tho College of Medicine and Pharmacy, No. 2201 Hung Vuong, Gia Cam, Viet Tri, Phu Tho, Vietnam \\ ${ }^{2}$ School of Chemical Engineering, Hanoi University of Science and Technology, No. 1 Dai Co Viet, Hai Ba Trung, Hanoi, Vietnam \\ Correspondence should be addressed to Tran Khac Vu; vutrankhac68@gmail.com
}

Received 2 May 2021; Revised 7 June 2021; Accepted 11 July 2021; Published 19 July 2021

Academic Editor: Nenad Ignjatovic

Copyright (c) 2021 Dao Viet Hung and Tran Khac Vu. This is an open access article distributed under the Creative Commons Attribution License, which permits unrestricted use, distribution, and reproduction in any medium, provided the original work is properly cited.

\begin{abstract}
The protein kinase Pkc1 of Candida albicans ( $\mathrm{CaPkc1})$, one of the key proteins involved in MAPK pathway, is described as a regulator of cell wall integrity during growth, morphogenesis, and response to cell wall stress. The (-)-cercosporamide is an antifungal natural product isolated from the phytopathogen fungus Cercosporidium henningsii. This phytoxin was found to inhibit selectively $\mathrm{CaPkcl}$ and constitutes an interesting model for the design of novel antifungal molecules. In this research, 4,7,9trihydroxy[1] benzofuro[3,2-d]pyrimidine-6-carboxamide (13) derived from (-)-cercosporamide was synthesized via a seven-step procedure by well-known reactions and evaluation of cytotoxicity and inhibition of CaPkc1. The bioassay showed CaPkc1 inhibitory activity $87 \%$ higher and cytotoxicity 100 times less than the reference, (-)-cercosporamide.
\end{abstract}

\section{Introduction}

Candidiasis exists in all regions of the world; however, there are significant variations between regions and it is difficult to give an average incidence of candidiasis. Although many studies have been carried out annually, the type of population recruited is variable and the results rendered are difficult to compare. In addition, it is absolutely necessary to know the profile of the local epidemiology in order to best adapt the antifungal treatment. American studies report that the annual incidence of Candida infections is 8 per 100,000 inhabitants $[1,2]$. In Europe, Candida spp. are responsible for 2-3\% of septicemia ranking between 6th and 10th among pathogens most frequent [3]. The distribution of Candida species in patients with candidiasis has changed over the past 20 years. The incidence of Candida albicans decreased (from $70 \%$ to $50 \%$ ), while the incidence of non-albicans Candida (NAC) species, including C. glabrata, C. krusei, C. tropicalis, C. auris, and C. parapsilosis has increased [4]. Until recently, C. albicans remains mainly responsible for these invasive infections, but the distribution of NAC species evolves and differs according to the regions of the world [5]. In C. albicans, protein kinase $\mathrm{C}(\mathrm{CaPkc})$ is one of the proteins involved in the MAPK pathway [6]. It is described as a regulator of the integrity of the cell wall during growth, morphogenesis, and the response to cell wall stress [7]. Treatment of fungal infections is very difficult because of limited therapeutic arsenal and emergence of resistance to the two antifungal major classes: azoles and echinocandins. Consequently, the search for new targets and new therapeutic strategies is a priority. In this context, targeting PKCmediated signal transduction pathway represents a new attractive strategy for antifungal therapy. The (-)-cercosporamide is an antifungal natural product isolated from the phytopathogen fungus Cercosporidium henningsii [8]. This phytoxin was found to inhibit selectively Pkcl kinase of C. albicans $\left(\mathrm{IC}_{50}=44 \mathrm{nM}\right)[9]$ and constitutes an interesting model for the design of novel antifungal molecules. In addition, research results suggest dihydroxybenzofurancarboxamide ring as the pharmacophore of the molecule, 
justifying the strategy for the design of new tricyclic compounds [10]. Regarding this, our expertise in the synthesis of pyrimidine-fused heterocycles as biological agents targeting kinases was used for the design of the new compounds, constituting the third ring of the benzofuro[3,2-d]pyrimidine derivatives described [11]. In this study, we designed and synthesized a new compound 13, which remained the dihydroxybenzofuran-carboxamide ring (part A, excluding asymmetric carbon), replacing part B with pyrimidine ring (Figure 1) targeting $\mathrm{CaPkcl}$. The introduction of a pyrimidine ring into the structure is capable of providing donor or acceptor groups for hydrogen bonds and can cause additional interactions with amino acids at the active site for better kinase inhibitory activity and improved selectivity.

\section{Results and Discussion}

2.1. Chemistry. As shown in Figure 2, analogues of (-)-cercosporamide were prepared from 3,5-dimethoxyphenol (1). Diiodination of compound 1 was performed very quickly at low temperature in the presence of NIS, followed by O-alkylation with a base and ethyl bromoacetate $[12,13]$ to afford 3 in $72 \%$. Subsequent cyanation of this intermediate in the presence of cuprous cyanide in DMF gave ester 4 in 75\% [14]. Cyclization using sodium hydride furnished benzofuran ring bearing the amino group at position 3 and ester function at position 2 [15]. Tricyclic pyrimidine derivative $\mathbf{6}$ was synthesized from benzofuran precursor 5 [16]. From compound 6, attempts for the conversion of nitrile to carboxamide remained unsuccessful [13, 17-19].

Another strategy was to introduce the carboxamide group before the cyclization step. As shown in Figure 3, a direct aminocarbonylation of compound $\mathbf{1}$ use chlorosulfonylisocyanate (CSI) as electrophilic reagent afforded benzamide derivative 8 after acidic hydrolysis of the corresponding $\mathrm{N}$-chlorosulfonyl carboxamide intermediate [20]. Next, the iodination of compound $\mathbf{8}$ was performed very quickly at $0^{\circ} \mathrm{C}$ in the presence of NIS, followed by cyanation in the presence of cuprous cyanide in DMF, and then, O-alkylation with a base and ethyl bromoacetate gave ester 11 in $90 \%$. Afterwards, the same reaction sequence as previously described in Scheme 1 was applied to obtain derivative 7 bearing the carbamoyl appendage at C-6 position of the azaheterocycle. Finally, demethylation of the methoxy groups was carried out by heating in neat pyridine hydrochloride at $200^{\circ} \mathrm{C}$ under microwave irradiation to provide the desired 4,7,9-trihydroxy[1]benzofuro[3, 2-d] pyrimidine-6-carboxamide (13) in 43\% [21]. The structures of all compounds synthesized were confirmed using MS, ${ }^{1} \mathrm{H}$, and ${ }^{13} \mathrm{C}$ NMR spectrum (Supplementary Materials).

2.2. Biological Activity. The inhibition of PKC activity $(\mathrm{CaPkc1})$ was initially investigated in order to check the involvement of this protein as the putative target of $4,7,9$ trihydroxy[1] benzofuro[3,2-d]pyrimidine-6-carboxamide

(13). Pkc activity of C. albicans (CAAL93 sensitive to fluconazole); total protein extracts was measured using ELISA-

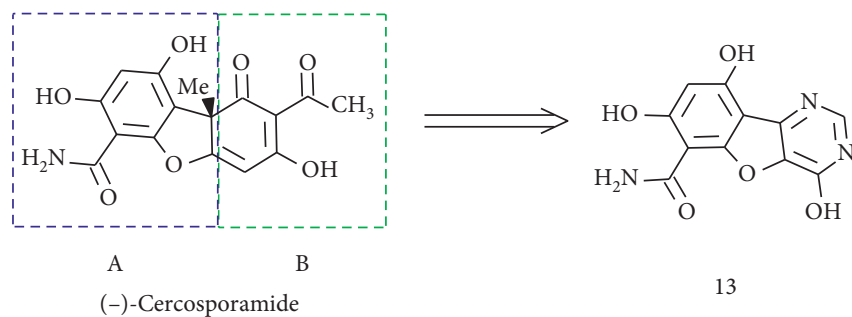

FiguRE 1: Design of target compound 13.

based PKC Kinase Activity Assay (Enzo Life Sciences, Inc.) [22]. PKC inhibition activity was expressed as the ratio between the absorbance measured for protein extract treated with compounds $13(100 \mu \mathrm{M})$ and untreated protein extract. The reference natural (-)-cercosporamide was provided by extraction and purification from a Mycosphaerella henningsii culture using a modified procedure of the literature $[8,23]$.

Mean percentage of inhibition of PKC activity was obtained for each compound $(100 \mu \mathrm{M})$ and compared with mean results obtained for $(-)$-cercosporamide $(100 \mu \mathrm{M})$ applying a one-way ANOVA followed by multiple comparisons with an uncorrected Fisher's LSD test. $P$ values were expressed for the uncorrected Fisher's LSD test $(p<0.00001)$. To investigate in vitro cytotoxic activity of 4,7,9-trihydroxy[1]benzofuro[3,2-d]pyrimidine-6-carboxamide (13), assay on HeLa cells was performed. As given in Table 1, compound 13 proved to be at least 100 times less cytotoxic than (-)-cercosporamide. This shows that compound $\mathbf{1 3}$ has the potential to become a new selective effect on fungal cells, not toxic to other cells. As depicted in Table 1 , when used at $100 \mu \mathrm{M},(-)$-cercosporamide displayed a moderate Pkc inhibitory activity of $53 \pm 3 \%$ in our conditions. Interestingly, compound $\mathbf{1 3}$ showed statistically $(p<0.05)$ significant higher inhibition with values of $87 \pm 2 \%$ at $100 \mu \mathrm{M}$. It is possible that the pyrimidine ring in part $\mathrm{B}$ in the structure has an additional $-\mathrm{OH}$ group resulting in stronger binding capacity of compound $\mathbf{1 3}$ to the center associated with $\mathrm{CaPkcl}$, increasing the ability to inhibit $\mathrm{CaPkcl}$ of compound 13.

\section{Conclusions}

An efficient and practically feasible procedure for the synthesis of 4,7,9-trihydroxy[1]benzofuro[3,2-d]pyrimidine-6carboxamide via a seven-step procedure using common chemical reactions such as direct aminocarbonylation, iodonation, cyanation, O-alkylation, cyclizaction, and demethylation has been described. In this research, the initial biological assay of compound 13 suggests further chemical modifications to evaluate the structure-activity relationship of this naturally derived potential compound, (-)-cercosporamide.

\section{Experimental}

All reactions were monitored by TLC analysis using Merck silicagel 60F-254 thin-layer plates. Column chromatography was carried out on silicagel Merck 60 (70-230 mesh ASTM). 

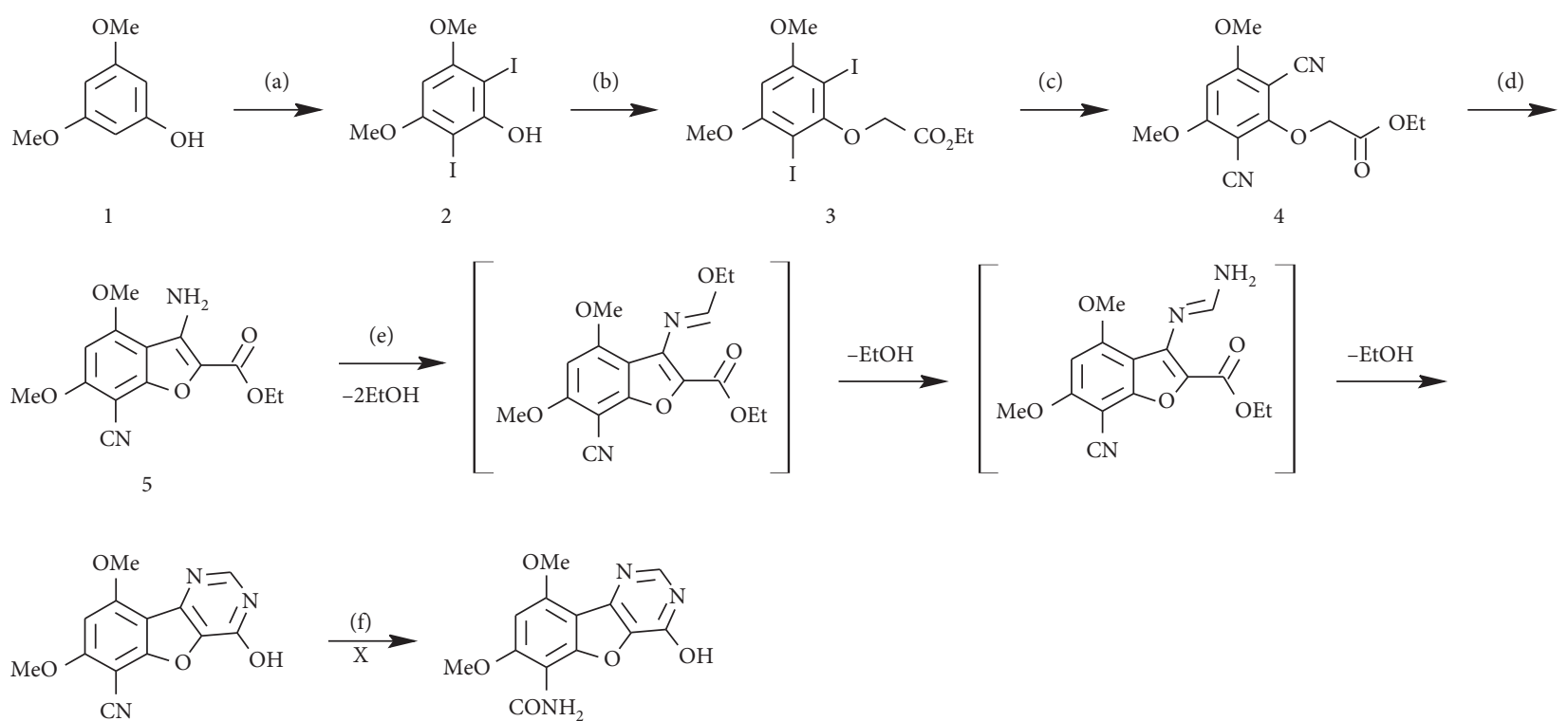

6

7

Figure 2: (a) NIS, $\mathrm{CH}_{2} \mathrm{Cl}_{2},-78^{\circ} \mathrm{C}, 2 \mathrm{~min}, 71 \%$. (b) $\mathrm{NaH} 60 \%$, DMF, ethyl bromoacetate, rt, $12 \mathrm{~h}, 72 \%$. (c) CuCN, DMF, $160^{\circ} \mathrm{C}, 2 \mathrm{~h}, 75 \%$. (d) NaH $60 \%$, DMF, $0^{\circ} \mathrm{C}$, $30 \mathrm{~min}, 51 \%$. (e) (1) $\mathrm{HC}(\mathrm{OEt})_{3}$, MW, $200^{\circ} \mathrm{C}, 15 \mathrm{~min}$, (2) $\mathrm{NH}_{3} / \mathrm{MeOH} 7 \mathrm{~N}, \mathrm{MW}, 140^{\circ} \mathrm{C}, 15 \mathrm{~min}, 46 \%$. (f) $\mathrm{H}_{2} \mathrm{O}_{2} 35 \%, \mathrm{~K}_{2} \mathrm{CO}, \mathrm{rt}_{3}, 24 \mathrm{~h}$ or $\mathrm{NaOH} / \mathrm{EtOH} 6 \mathrm{~N}, \mathrm{H}_{2} \mathrm{O}_{2} 35 \%$, rt, $24 \mathrm{~h}$ or urea-hydrogen peroxide, $\mathrm{K}_{2} \mathrm{CO}_{3}$, acetone/ $\mathrm{H}_{2} \mathrm{O}$, rt, $5 \mathrm{~h}$ or $\mathrm{BF}_{3} .2 \mathrm{CH}_{3} \mathrm{COOH}_{\text {or }} \mathrm{H}_{2} \mathrm{SO}_{4} \mathrm{cc}, 15 \mathrm{~min}$, failure.<smiles>COc1cc(O)cc(OC)c1</smiles>

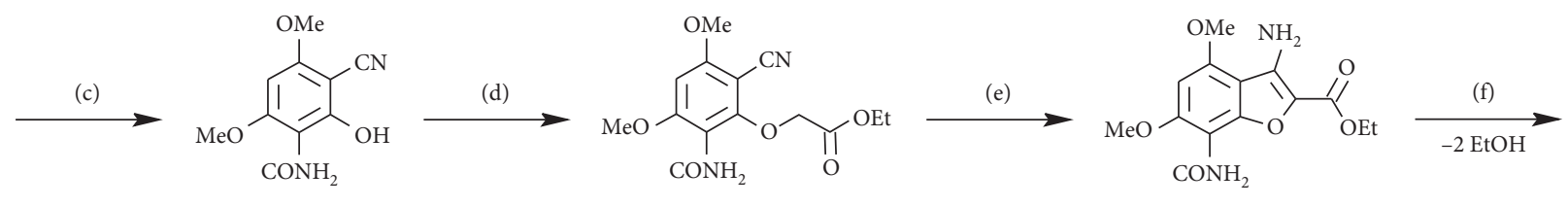<smiles>CCOC=Nc1c(C(=O)OCC)oc2c(C(=O)O)c(OC)cc(OC)c12</smiles><smiles>NC(=O)c1c(O)cc(O)c2oc3c(O)ncnc3c12</smiles>

13

Figure 3: (a) CSI, $\mathrm{CH}_{3} \mathrm{CN}, 0^{\circ} \mathrm{C}, 10 \mathrm{~min}$ and then $\mathrm{HCl} 5 \mathrm{M}, \mathrm{rt}, 10 \mathrm{~h}, 45 \%$. (b) NIS, $\mathrm{CH}_{2} \mathrm{Cl}_{2}, 0^{\circ} \mathrm{C}, 10 \mathrm{~min}, 60 \%$. (c) $\mathrm{CuCN}, \mathrm{DMF}, 160^{\circ} \mathrm{C}, 6 \mathrm{~h}, 38 \%$. (d) $\mathrm{NaH}, \mathrm{BrCH}_{2} \mathrm{CO}_{2} \mathrm{Et}, \mathrm{DMF}, \mathrm{rt}, 24 \mathrm{~h}, 90 \%$. (e) $\mathrm{NaH}, \mathrm{DMF}, 0^{\circ} \mathrm{C}, 10 \mathrm{~min}, 67 \%$. (f) $\mathrm{HC}(\mathrm{OEt})_{3}, \mathrm{MW}, 200^{\circ} \mathrm{C}, 15 \mathrm{~min}$ and then $\mathrm{NH}_{3} / \mathrm{MeOH}^{2} \mathrm{~N}$, $\mathrm{MW}, 140^{\circ} \mathrm{C}, 15 \mathrm{~min}, 62 \%$. (g) Pyridine. $\mathrm{HCl}, \mathrm{MW}, 200^{\circ} \mathrm{C}, 10 \mathrm{~min}, 43 \%$.

Melting points were determined on an Electrothermal IA 9000 melting point apparatus and are uncorrected. Infrared spectra (IR) were recorded on a Shimadzu IRAffinity-1 IR-
FT spectrophotometer equipped with a MIRacle 10 accessory ATR. ${ }^{1} \mathrm{H}$ and ${ }^{13} \mathrm{C}$ NMR spectra were performed in DMSO- $\mathrm{d}_{6}$ or $\mathrm{CDCl}_{3}$ using a Bruker Avance $400 \mathrm{MHz}$ spectrometer. 
TABLE 1: Activity of compound $\mathbf{1 3}$ on protein extract of CAAL93 and HeLa.

$\begin{array}{ll}\text { Compounds } & \text { MIC values }(\mu \mathrm{M})^{\mathrm{a}} \\ \text { Protein extract of CAAL93 }\end{array}$

${ }^{\mathrm{a}}$ Values represent the mean \pm SD of experiments performed in triplicate. MIC was determined as the compound concentration that produced $50 \%$ of growth inhibition relative to that of the drug-free growth control. ${ }^{\mathrm{b}} \mathrm{CERCO},(-)$-cercosporamide.

Chemical shifts are reported as $\delta$ values in parts per million (ppm) relative to tetramethylsilane as internal standard, and coupling constants $(\mathrm{J})$ are given in $\operatorname{Hertz}(\mathrm{Hz})$. The following abbreviations are used to describe peak patterns when appropriate: s (singlet), d (doublet), $t$ (triplet), q (quartet), and $\mathrm{m}$ (multiplet). Mass spectra were recorded using an electrospray ionization method with the Waters ZQ 2000 spectrometer. Microwave-promoted reactions were performed on a CEM Discover SP monomode apparatus. Elemental analyses were performed on a Thermo Scientific Elemental Analyser Flash EA 1112 and were found within $\pm 0.4 \%$ of the theoretical values.

4.1. 2,6-Diiodo-3,5-dimethoxyphenol (2). A solution of 3,5dimethoxyphenol (1) (1.00 g, $6.50 \mathrm{mmol})$ in dichloromethane $(20 \mathrm{~mL})$ was cooled to $-78^{\circ} \mathrm{C}$. $N$-Iodosuccinimide $(3.22 \mathrm{~g}, 14.3 \mathrm{mmol})$ was added, and the reaction was maintained at $-78^{\circ} \mathrm{C}$ for $2 \mathrm{~min}$. The reaction was quenched at $-78^{\circ} \mathrm{C}$ by the addition of $10 \%$ aqueous potassium carbonate solution $(20 \mathrm{~mL})$ and warmed to room temperature. The reaction was diluted with water $(50 \mathrm{~mL})$ and extracted with dichloromethane $(3 \times 50 \mathrm{~mL})$. The combined organic extracts were dried over sodium sulfate and filtered. The solvent was evaporated in vacuo, and the residue was purified by silica gel column chromatography using cyclohexane/ethyl acetate $(9 / 1)$ as an eluent system to give compound 2 as a beige solid $(1.87 \mathrm{~g}, 71 \%)$. $R_{\mathrm{f}}=0.32$ (cyclohexane/ethyl acetate: $8 / 2) ; \mathrm{Mp}=112-113^{\circ} \mathrm{C}$. IR $\left(\mathrm{cm}^{-1}\right)$ : 3423, 1496, 1446, 1379, 1336, 1292, 1184, 1093, 1064, 1033, 804, 729, 692. ${ }^{1} \mathrm{H}$ NMR $\left(400 \mathrm{MHz}, \mathrm{CDCl}_{3}\right) \delta: 6.05(\mathrm{~s}, 1 \mathrm{H}$, $\mathrm{H}-4), 5.94(\mathrm{~s}, 1 \mathrm{H}, \mathrm{OH}), 3.92\left(\mathrm{~s}, 6 \mathrm{H}, 2 \mathrm{CH}_{3}\right) .{ }^{13} \mathrm{C} \mathrm{NMR}$ $\left(100 \mathrm{MHz}, \mathrm{CDCl}_{3}\right) \delta: 158.9$ (2C-3,5), 155.0 (C-1), $91.8(\mathrm{C}-4)$, $65.9(2 \mathrm{C}-2,6) 56.4\left(2 \mathrm{CH}_{3}\right)$. MS (ESI), $m / z(\%): 406.1(100)$ $[\mathrm{M}+\mathrm{H}]^{+}$.

4.2. Ethyl (2,6-diiodo-3,5-dimethoxyphenoxy) acetate (3). To a solution of 2,6-diiodo-3,5-dimethoxyphenol (2) (1.00 g, $2.46 \mathrm{mmol})$ in $N, N$-dimethylformamide $(3 \mathrm{~mL})$ was added sodium hydride $60 \%(148 \mathrm{mg}, 3.70 \mathrm{mmol})$ at room temperature. When no more gas evolved, ethyl bromoacetate $(0.4 \mathrm{~mL}, 3.70 \mathrm{mmol})$ was added dropwise, and the reaction mixture was stirred at room temperature for $12 \mathrm{~h}$. After addition of water, the aqueous layer was extracted with dichloromethane $(3 \times 50 \mathrm{~mL})$. The combined organic extracts were dried over sodium sulfate and filtered. The solvent was evaporated in vacuo, and the residue was purified by silica gel column chromatography using cyclohexane/ethyl acetate $(9 / 1)$ as an eluent system to give compound 3 as a white solid (871 mg, 72\%). $R_{f}=0.27$ (cyclohexane/ethyl acetate: $8 / 2) . \mathrm{Mp}=112-113^{\circ} \mathrm{C}$. IR $\left(\mathrm{cm}^{-1}\right)$ : $1751,1566,1334,1107,738 .{ }^{1} \mathrm{H}$ NMR $\left(400 \mathrm{MHz}, \mathrm{CDCl}_{3}\right) \delta$ : 6.32 (s, 1H, H-4), 4.61 (s, 2H, $\left.\mathrm{OCH}_{2}\right), 4.35$ (q, J=7.2 Hz, $2 \mathrm{H}$, $\mathrm{CH}_{2} \mathrm{CH}_{3}$ ), $3.91\left(\mathrm{~s}, 6 \mathrm{H}, 2 \mathrm{CH}_{3}\right), 1.37\left(\mathrm{t}, J=7.1 \mathrm{~Hz}, 3 \mathrm{H}, \mathrm{CH}_{3}\right)$. ${ }^{13} \mathrm{C} \mathrm{NMR}\left(100 \mathrm{MHz}, \mathrm{CDCl}_{3}\right) \delta: 167.7(\mathrm{C}=\mathrm{O}), 159.3(2 \mathrm{C}-3,5)$, 158.2 (C-1), 95.9 (C-4), 73.6 (2C-2,6), $68.7\left(\mathrm{CH}_{2} \mathrm{CO}\right), 61.3$ $\left(\mathrm{CH}_{2} \mathrm{CH}_{3}\right), 56.2\left(2 \mathrm{CH}_{3}\right), 14.2\left(\mathrm{CH}_{3}\right) . \mathrm{MS}(\mathrm{ESI}), \mathrm{m} / z(\%)$ : $493.1(100)[\mathrm{M}+\mathrm{H}]^{+}$.

4.3. Ethyl (2,6-dicyano-3,5-dimethoxyphenoxy) acetate (4). A mixture of ethyl (2,6-diiodo-3,5-dimethoxyphenoxy) acetate (3) (800 mg, $1.63 \mathrm{mmol})$ and copper (I) cyanide (394 mg, $4.4 \mathrm{mmol}$ ) in $N, N$-dimethylformamide $(3 \mathrm{~mL})$ was heated at $160^{\circ} \mathrm{C}$ for $2 \mathrm{~h}$. After cooling, the reaction mixture was poured into crushed ice, and a precipitate was formed, which was extracted with ethyl acetate $(2 \times 30 \mathrm{~mL})$. The organic layers were washed with brine, dried over sodium sulfate, filtered, and evaporated to dryness. The resulting oil was purified by silica gel column chromatography using cyclohexane/ethyl acetate $(8 / 2)$ as eluent to give compound 4 as a white solid (355 mg, 75\%). $R_{f}=0.60$ (cyclohexane/ethyl acetate: $6 / 4) ; \mathrm{Mp}=65-66^{\circ} \mathrm{C}$. IR $\left(\mathrm{cm}^{-1}\right): 2924,2222,1755$, 1597, 1498, 1381, 1176, 1122. ${ }^{1} \mathrm{H}$ NMR $\left(400 \mathrm{MHz}, \mathrm{CDCl}_{3}\right) \delta$ : $6.26(\mathrm{~s}, 1 \mathrm{H}, \mathrm{H}-4), 5.13\left(\mathrm{~s}, 2 \mathrm{H}, \mathrm{COCH}_{2}\right), 4.28$ (q, $2 \mathrm{H}$, $\left.J=7.1 \mathrm{~Hz}, \mathrm{CH}_{2} \mathrm{CH}_{3}\right), 3.91\left(\mathrm{~s}, 6 \mathrm{H}, 2 \mathrm{CH}_{3}\right), 1.30(\mathrm{t}, 3 \mathrm{H}$, $\left.J=7.1 \mathrm{~Hz}, \mathrm{CH}_{3}\right) .{ }^{13} \mathrm{C} \mathrm{NMR}\left(100 \mathrm{MHz}, \mathrm{CDCl}_{3}\right) \delta: 167.3$ $(\mathrm{C}=\mathrm{O}), 165.4(2 \mathrm{C}-3,5), 164.4(\mathrm{C}-1), 112.5(2 \mathrm{C} \equiv \mathrm{N}), 93.1(\mathrm{C}-$ 4), 88.7 (2C-2,6), $69.1\left(\mathrm{CH}_{2} \mathrm{CO}\right), 61.9\left(\mathrm{CH}_{2} \mathrm{CH}_{3}\right), 56.4$ $\left(2 \mathrm{CH}_{3}\right), 14.0\left(\mathrm{CH}_{3}\right)$. MS (ESI), $\mathrm{m} / z \quad(\%): 291.1$ (100) $[\mathrm{M}+\mathrm{H}]^{+}$.

4.4. Ethyl 3-amino-7-cyano-4,6-dimethoxy-1-benzofuran2-carboxylate (5). To a solution of ethyl (2,6-dicyano-3,5dimethoxyphenoxy) acetate (4) (300 mg, $1.03 \mathrm{mmol}$ ) in $N, N$ dimethylformamide $(3 \mathrm{~mL})$ was added sodium hydride $60 \%$ 
(84 mg, $2.1 \mathrm{mmol}$ ) at $0^{\circ} \mathrm{C}$. The mixture was stirred for $30 \mathrm{~min}$, quenched with water, and extracted with ethyl acetate $(100 \mathrm{~mL})$. The organic layers were washed with brine, dried over sodium sulfate, filtered, and evaporated to dryness. The resulting oil was purified by silica gel column chromatography using cyclohexane/ethyl acetate (8/2) as an eluent system to give compound 5 as a beige solid $(153 \mathrm{mg}, 51 \%$ yield). $\quad R_{f}=0.71 \quad$ (cyclohexane/ethyl acetate: 1/1); $\mathrm{Mp}=124-125^{\circ} \mathrm{C}$. IR $\left(\mathrm{cm}^{-1}\right): 2223,1614,1554,1508,1454$, $1338,1305,1103,966,700 .{ }^{1} \mathrm{H}$ NMR (400 MHz, DMSO-d 6 ), $\delta$ ppm: 6.98 (s, 1H, H-5), $5.90\left(\mathrm{~s}, 2 \mathrm{H}, \mathrm{NH}_{2}\right), 4.32(\mathrm{q}, 2 \mathrm{H}$, $\left.J=7.1 \mathrm{~Hz}, \mathrm{CH}_{2} \mathrm{CH}_{3}\right), 3.91\left(\mathrm{~s}, 6 \mathrm{H}, 2 \mathrm{CH}_{3}\right), 1.34(\mathrm{t}, 3 \mathrm{H}$, $\left.J=7.1 \mathrm{~Hz}, \mathrm{CH}_{3}\right) .{ }^{13} \mathrm{C}$ NMR $\left(100 \mathrm{MHz}\right.$, DMSO-d $\left.\mathrm{d}_{6}\right), \delta \mathrm{ppm}$ : $163.9(\mathrm{C}=\mathrm{O}), 160.8(\mathrm{C}-6), 158.6(\mathrm{C}-4), 155.4(\mathrm{C}-3), 124.6$ (C2), $112.6(\mathrm{C} \equiv \mathrm{N}), 106.3(\mathrm{C}), 92.0(\mathrm{C}-5), 80.5(\mathrm{C}-7), 60.1$ $\left(\mathrm{CH}_{2} \mathrm{CH}_{3}\right), 56.7\left(\mathrm{CH}_{3}\right), 56.0\left(\mathrm{CH}_{3}\right), 53.4(\mathrm{C}), 14.6\left(\mathrm{CH}_{3}\right) . \mathrm{MS}$ (ESI), $m / z(\%): 291.2(100)[\mathrm{M}+\mathrm{H}]^{+}$.

4.5. 4-Hydroxy-7,9-dimethoxy[1]benzofuro[3,2-d]pyrimidine6-carbonitrile (6). A mixture of ethyl 3-amino-7-cyano4,6-dimethoxy-1-benzofuran-2-carboxylate (5) (100 mg, $0.34 \mathrm{mmol})$ in triethyl orthoformate $(2 \mathrm{~mL})$ was irradiated in a microwave at $200^{\circ} \mathrm{C}$ for $15 \mathrm{~min}$. The resultant yellow solution was evaporated to dryness, and the residue was dissolved in a solution of ammonia in methanol $7 \mathrm{~N}$ $(2 \mathrm{~mL})$. The mixture was irradiated in a microwave at $140^{\circ} \mathrm{C}$ for $15 \mathrm{~min}$, and the resultant precipitate was collected and evaporated to dryness. The residue was triturated with acetonitrile, and the solid was collected by filtration. The solids were combined to give compound $\mathbf{6}$ as a white solid (43 mg, 46\%). $R_{f}=0.65$ (ethyl acetate); $\mathrm{Mp}=268-269^{\circ} \mathrm{C}$. IR $\left(\mathrm{cm}^{-1}\right): 3182,2222,1685,1598,1487,1433,1366,1116,1093$, 1022, 962, 692. ${ }^{1} \mathrm{H}$ NMR $\left(400 \mathrm{MHz}, \mathrm{DMSO}-\mathrm{d}_{6}\right) \delta: 13.03(\mathrm{~s}$, $1 \mathrm{H}, \mathrm{OH}), 8.27$ (s, $1 \mathrm{H}, \mathrm{H}-2), 7.20$ (s, $1 \mathrm{H}, \mathrm{H}-8), 4.05$ (s, $3 \mathrm{H}$, $\left.\mathrm{CH}_{3}\right), 3.93\left(\mathrm{~s}, 3 \mathrm{H}, \mathrm{CH}_{3}\right) .{ }^{13} \mathrm{C}$ NMR $\left(100 \mathrm{MHz}, \mathrm{DMSO}_{6} \mathrm{~d}_{6}\right) \delta$ : 164.1 (C-4), 159.2 (C-9), 155.9 (C-7), 154.6 (C), 153.3 (C), $147.6(\mathrm{C}-2), 145.3(\mathrm{C}), 112.6(\mathrm{C} \equiv \mathrm{N}), 105.0(\mathrm{C}-6), 104.9(\mathrm{C})$, 91.9 (C-8), $56.6\left(\mathrm{CH}_{3}\right), 56.1\left(\mathrm{CH}_{3}\right)$. MS (ESI), $m / z(\%): 272.1$ (100) $[\mathrm{M}+\mathrm{H}]^{+}$.

4.6. 2-Hydroxy-4,6-dimethoxybenzamide (8). To a stirred suspension of 3,5-dimethoxyphenol (1) ( $4.00 \mathrm{~g}, 26.00 \mathrm{mmol})$ in acetonitrile $(40 \mathrm{~mL})$ at $0^{\circ} \mathrm{C}$ under argon was added chlorosulfonyl isocyanate $(3.4 \mathrm{~mL}, 39.00 \mathrm{mmol})$. The mixture was maintained at this temperature for $10 \mathrm{~min}$; then, the reaction mixture was quenched with hydrochloric acid $5 \mathrm{M}$ $(40 \mathrm{~mL})$. After $10 \mathrm{~h}$ at room temperature, the reaction was diluted with water $(100 \mathrm{~mL})$ and extracted with dichloromethane $(3 \times 100 \mathrm{~mL})$. The combined organic extracts were dried over sodium sulfate and filtered. The solvent was evaporated in vacuo, and the residue was purified by silica gel column chromatography using cyclohexane/ethyl acetate $(8 / 2)$ as an eluent system to give compound $\mathbf{8}$ as a white solid $(2.30 \mathrm{~g}, 45 \%) . R_{f}=0.46$ (cyclohexane/ethyl acetate: 6/4); $\mathrm{Mp}=154-155^{\circ} \mathrm{C}$. IR $\left(\mathrm{cm}^{-1}\right): 3429,3209,1620,1587,1492$, $1415,1355,1315,1294,1217,1199,1159,1109,1041 .{ }^{1} \mathrm{H}$ NMR (400 MHz, DMSO-d $\left.\mathrm{d}_{6}\right) \delta: 14.69(\mathrm{~s}, 1 \mathrm{H}, \mathrm{OH}), 8.00(\mathrm{~d}$, $\left.J=3.20 \mathrm{~Hz}, 2 \mathrm{H}, \mathrm{CONH}_{2}\right), 6.08(\mathrm{~d}, J=2.50 \mathrm{~Hz}, 1 \mathrm{H}, \mathrm{H}-5), 6.05$ $(\mathrm{d}, J=2.40 \mathrm{~Hz}, 1 \mathrm{H}, \mathrm{H}-3), 3.87\left(\mathrm{~s}, 3 \mathrm{H}, \mathrm{CH}_{3}\right), 3.77(\mathrm{~s}, 3 \mathrm{H}$, $\left.\mathrm{CH}_{3}\right) .{ }^{13} \mathrm{C}$ NMR $\left(100 \mathrm{MHz}\right.$, DMSO-d $\left.\mathrm{d}_{6}\right) \delta: 171.7(\mathrm{C}=\mathrm{O})$, 165.9 (C-2), 163.7 (C-6), 160.2 (C-4), 96.6 (C-1), 94.1 (C-3), 90.1 (C-5), $56.0\left(\mathrm{CH}_{3}\right), 55.3\left(\mathrm{CH}_{3}\right)$. MS (ESI), $m / z(\%): 198.1$ (100) $[\mathrm{M}+\mathrm{H}]^{+}$.

4.7. 2-Hydroxy-3-iodo-4,6-dimethoxybenzamide (9). A solution of 2-hydroxy-4,6-dimethoxybenzamide (8) (2.00 g, $10.15 \mathrm{mmol})$ in dichloromethane $(40 \mathrm{~mL})$ at $0^{\circ} \mathrm{C}$ under argon was added $\mathrm{N}$-iodosuccinimide $(2.28 \mathrm{~g}, 10.15 \mathrm{mmol})$, and the reaction mixture was maintained at this temperature for $10 \mathrm{~min}$. The reaction was then diluted with water $(60 \mathrm{~mL})$ and extracted with dichloromethane $(3 \times 100 \mathrm{~mL})$. The combined organic extracts were dried over sodium sulfate and filtered. The solvent was evaporated in vacuo, and the residue was purified by silica gel column chromatography using cyclohexane/ethyl acetate $(6 / 4)$ as an eluent system to give compound 9 as a white solid $(1.97 \mathrm{~g}, 60 \%) . R_{f}=0.21$ (cyclohexane/ethyl acetate: 6/4). $\mathrm{Mp}=270-271^{\circ} \mathrm{C} . \operatorname{IR}\left(\mathrm{cm}^{-1}\right)$ : $3439,1602,1579,1415,1396,1336,1286,1215,1120,1078^{1} \mathrm{H}$ NMR $\left(400 \mathrm{MHz}\right.$, DMSO-d $\left.\mathrm{d}_{6}\right) \delta: 15.74(\mathrm{~s}, 1 \mathrm{H}, \mathrm{OH}), 8.13(\mathrm{~d}$, $\left.J=1.2 \mathrm{~Hz}, 2 \mathrm{H}, \mathrm{CONH}_{2}\right), 6.30(\mathrm{~s}, 1 \mathrm{H}, \mathrm{H}-5), 3.97\left(\mathrm{~s}, 3 \mathrm{H}, \mathrm{CH}_{3}\right)$, $3.92\left(\mathrm{~s}, 3 \mathrm{H}, \mathrm{CH}_{3}\right) .{ }^{13} \mathrm{C}$ NMR $(100 \mathrm{MHz}, \mathrm{DMSO}) \delta: 171.3$ $(\mathrm{C}=\mathrm{O}), 163.9$ (C-2), 162.1 (C-6), $161.2(\mathrm{C}-4), 97.1(\mathrm{C}-1), 87.5$ (C-5), $67.1(\mathrm{C}-3), 56.5\left(\mathrm{CH}_{3}\right), 56.4\left(\mathrm{CH}_{3}\right)$. MS (ESI), $\mathrm{m} / z(\%)$ : $324.0(100)[\mathrm{M}+\mathrm{H}]^{+}$.

4.8. 3-Cyano-2-hydroxy-4,6-dimethoxybenzamide (10). A mixture of 2-hydroxy-3-iodo-4,6-dimethoxybenzamide (9) $(1.00 \mathrm{~g}, 3.10 \mathrm{mmol})$ and copper (I) cyanide $(465 \mathrm{mg}$, $5.20 \mathrm{mmol}$ ) in $\mathrm{N}, \mathrm{N}$-dimethylformamide $(4 \mathrm{~mL})$ was heated at $160^{\circ} \mathrm{C}$ for $6 \mathrm{~h}$. After cooling, the reaction mixture was poured into crushed ice, and a precipitate was formed, which was extracted with ethyl acetate $(100 \mathrm{~mL})$. The organic layers were washed with brine, dried over sodium sulfate, filtered, and evaporated to dryness. The resulting oil was purified by silica gel column chromatography using dichloromethane/ methanol (9/1) as an eluent system to give compound $\mathbf{1 0}$ as a beige solid $(262 \mathrm{mg}, 38 \%) . \quad R_{\mathrm{f}}=0.82$ (dichloromethane/ methanol: $9 / 1) . \mathrm{Mp}=300-301^{\circ} \mathrm{C} . \mathrm{IR}\left(\mathrm{cm}^{-1}\right): 3477,3367$, 2220, 1651, 1583, 1483, 1450, 1427, 1344, 1209, 1126, 1070. ${ }^{1} \mathrm{H}$ NMR $\left(400 \mathrm{MHz}\right.$, DMSO-d $\left.\mathrm{d}_{6}\right) \delta: 16.21(\mathrm{~s}, 1 \mathrm{H}, \mathrm{OH}), 8.34(\mathrm{~d}$, $\left.J=6.4 \mathrm{~Hz}, 2 \mathrm{H}, \mathrm{CONH}_{2}\right), 6.32(\mathrm{~s}, 1 \mathrm{H}, \mathrm{H}-5), 4.03\left(\mathrm{~s}, 3 \mathrm{H}, \mathrm{CH}_{3}\right)$, $3.98\left(\mathrm{~s}, 3 \mathrm{H}, \mathrm{CH}_{3}\right) .{ }^{13} \mathrm{C}$ NMR $\left(100 \mathrm{MHz}, \mathrm{DMSO}-\mathrm{d}_{6}\right) \delta: 171.0$ $(\mathrm{C}=\mathrm{O}), 168.4(\mathrm{C}-4), 165.4(\mathrm{C}-6), 164.3(\mathrm{C}-2), 113.8(\mathrm{C} \equiv \mathrm{N})$, $96.5(\mathrm{C}-1), 87.4(\mathrm{C}-5), 82.8(\mathrm{C}-3), 57.0\left(\mathrm{CH}_{3}\right), 56.7\left(\mathrm{CH}_{3}\right)$. MS (ESI), $m / z$ (\%): $223.1(100)[\mathrm{M}+\mathrm{H}]^{+}$.

\subsection{Ethyl (2-carbamoyl-6-cyano-3,5-dimethoxyphenoxy)} acetate (11). To a solution of 3-cyano-2-hydroxy-4,6dimethoxybenzamide (10) $(222 \mathrm{mg}, 1.00 \mathrm{mmol})$ in $N, N-$ dimethylformamide $(2 \mathrm{~mL})$ was added sodium hydride $60 \%$ (48 $\mathrm{mg}, 1.20 \mathrm{mmol}$ ) at room temperature. When no more gas evolved, ethyl bromoacetate $(150 \mu \mathrm{L}, 1.40 \mathrm{mmol})$ was added, and the reaction mixture was stirred at room temperature for $24 \mathrm{~h}$. After addition of water, the aqueous layer was extracted with dichloromethane $(100 \mathrm{~mL})$. The combined 
organic extracts were dried over sodium sulfate and filtered. The solvent was evaporated in vacuo, and the residue was purified by silica gel column chromatography using cyclohexane/ethyl acetate $(8 / 2)$ as an eluent system to give compound 11 as a white solid $(277 \mathrm{mg}, 90 \%) . R_{f}=0.72$ (dichloromethane/methanol: 9/1). $M_{p}=85-86^{\circ} \mathrm{C}$. IR $\left(\mathrm{cm}^{-1}\right)$ : $3377,3186,2218,1645,1600,1562,1469,1390,1354,1332$, 1222, 1107, 1020. ${ }^{1} \mathrm{H}$ NMR (400 MHz, DMSO-d 6 ) $\delta: 7.83$ (s, $1 \mathrm{H}, \mathrm{CONH}_{2}$ ), 7.65 (s, 1H, $\left.\mathrm{CONH}_{2}\right), 6.89$ (s, 1H, H-4), 4.82 (s, $2 \mathrm{H}, \mathrm{CH}_{2} \mathrm{CO}$ ), 4.15 (q, J=7.10 Hz, $2 \mathrm{H}, \mathrm{CH}_{2} \mathrm{CH}_{3}$ ), 3.99 (s, $\left.3 \mathrm{H}, \mathrm{CH}_{3}\right), 3.92\left(\mathrm{~s}, 3 \mathrm{H}, \mathrm{CH}_{3}\right), 1.21\left(\mathrm{t}, J=7.10 \mathrm{~Hz}, 3 \mathrm{H}, \mathrm{CH}_{3}\right)$. ${ }^{13} \mathrm{C}$ NMR (100 MHz, DMSO-d 6 ) $\delta: 167.1(\mathrm{C}=\mathrm{O}), 164.2$ $(\mathrm{C}=\mathrm{O}), 161.8(\mathrm{C}-3), 160.3(\mathrm{C}-5), 157.6(\mathrm{C}-1), 114.8(\mathrm{C} \equiv \mathrm{N})$, 113.6 (C-2), 94.6 (C-4), 87.9 (C-6), $69.8\left(\mathrm{CH}_{2} \mathrm{CO}\right), 60.7$ $\left(\mathrm{CH}_{2} \mathrm{CH}_{3}\right), 57.0\left(\mathrm{CH}_{3}\right), 56.6\left(\mathrm{CH}_{3}\right), 13.9\left(\mathrm{CH}_{3}\right) . \mathrm{MS}(\mathrm{ESI}), \mathrm{m} /$ $z(\%): 309.2(100)[\mathrm{M}+\mathrm{H}]^{+}$.

4.10. Ethyl 3-amino-7-carbamoyl-4,6-dimethoxy-1-benzofuran2-carboxylate (12). To a solution of ethyl (2-carbamoyl6-cyano-3,5-dimethoxyphenoxy) acetate (11) (200 mg, $0.65 \mathrm{mmol})$ in $\mathrm{N}, \mathrm{N}$-dimethylformamide $(2 \mathrm{~mL})$ was added sodium hydride $60 \%$ in mineral oil $(25 \mathrm{mg}, 0.65 \mathrm{mmol})$ at $0^{\circ} \mathrm{C}$. The mixture was stirred for $10 \mathrm{~min}$, quenched with water, and extracted with ethyl acetate $(100 \mathrm{~mL})$. The organic layers were washed with brine, dried over sodium sulfate, filtered, and evaporated to dryness. The resulting oil was purified by silica gel column chromatography using cyclohexane/ethyl acetate $(6 / 4)$ as an eluent system to give compound 12 as a white solid $(134 \mathrm{mg}, 67 \%) . R_{f}=0.68$ (dichloromethane/methanol: 9/1). $M_{p}=215-216^{\circ} \mathrm{C}$. IR $\left(\mathrm{cm}^{-1}\right): 3462,3365,2922,1620,1556,1454,1296,1159,1105$, 1026. ${ }^{1} \mathrm{H}$ NMR $\left(400 \mathrm{MHz}, \mathrm{DMSO}-\mathrm{d}_{6}\right) \delta: 7.57$ (d, $J=5.2 \mathrm{~Hz}$, $2 \mathrm{H}, \mathrm{CONH}_{2}$ ), 6.54 (s, 1H, H-5), 6.22 (s, 2H, $\mathrm{NH}_{2}$ ), 4.26 (q, $\left.{ }^{3} J=7.10 \mathrm{~Hz}, 2 \mathrm{H}, \mathrm{CH}_{2} \mathrm{CH}_{3}\right), 3.97\left(\mathrm{~s}, 3 \mathrm{H}, \mathrm{CH}_{3}\right), 3.91(\mathrm{~s}, 3 \mathrm{H}$, $\left.\mathrm{CH}_{3}\right), 1.29\left(\mathrm{t},{ }^{3} \mathrm{~J}=7.10 \mathrm{~Hz}, 3 \mathrm{H}, \mathrm{CH}_{3}\right) .{ }^{13} \mathrm{C} \mathrm{NMR}(100 \mathrm{MHz}$, DMSO-d $\left.{ }_{6}\right) \delta: 164.7(\mathrm{C}=\mathrm{O}), 163.8(\mathrm{C}=\mathrm{O}), 159.4(\mathrm{C}-4), 156.0$ (C-6), 153.3 (C-2), 142.2 (C-3), 105.5 (C-7), 104.0 (C), 103.9 (C), $90.7(\mathrm{C}-5), 59.1\left(\mathrm{CH}_{2} \mathrm{CH}_{3}\right), 56.7\left(\mathrm{CH}_{3}\right), 56.0\left(\mathrm{CH}_{3}\right), 14.5$ $\left(\mathrm{CH}_{3}\right)$. MS (ESI), $m / z(\%): 309.2(100)[\mathrm{M}+\mathrm{H}]^{+}$.

4.11. 4-Hydroxy-7,9-dimethoxy[1]benzofuro[3,2-d]pyrimidine6-carboxamide (7). A mixture of ethyl 3-amino-7carbamoyl-4,6-dimethoxy-1-benzofuran-2-carboxylate (12) $(100 \mathrm{mg}, 0.32 \mathrm{mmol})$ in triethyl orthoformate $(2 \mathrm{~mL})$ was irradiated using microwave heating at $200^{\circ} \mathrm{C}$ for $15 \mathrm{~min}$. The resultant yellow solution was evaporated to dryness, and the residue was dissolved in a solution of ammonia in methanol $7 \mathrm{~N}(2 \mathrm{~mL})$. The mixture was then irradiated using microwave heating at $140^{\circ} \mathrm{C}$ for $15 \mathrm{~min}$, and the resultant precipitate was collected and evaporated to dryness. The residue was triturated with methanol, and the solid was collected by filtration. The solids were combined to give compound 7 as a brown solid (58 mg, 62\%). $R_{f}=0.21$ (dichloromethane/ methanol: 9/1). $\mathrm{Mp}=262-263^{\circ} \mathrm{C}$ (methanol). IR $\left(\mathrm{cm}^{-1}\right)$ : 3429, 3169, 1649, 1608, 1317, 1215, 1099, 1002. ${ }^{1} \mathrm{H}$ NMR $\left(400 \mathrm{MHz}, \mathrm{DMSO}-d_{6}\right) \delta: 8.31(\mathrm{~s}, 1 \mathrm{H}, \mathrm{H}-2), 7.76(\mathrm{~s}, 1 \mathrm{H}$, $\left.\mathrm{CONH}_{2}\right), 7.52\left(\mathrm{~s}, 1 \mathrm{H}, \mathrm{CONH}_{2}\right), 7.28(\mathrm{~s}, 1 \mathrm{H}, \mathrm{OH}), 6.71(\mathrm{~s}, 1 \mathrm{H}$, $\mathrm{H}-8), 4.05\left(\mathrm{~s}, 3 \mathrm{H}, \mathrm{CH}_{3}\right), 3.95\left(\mathrm{~s}, 3 \mathrm{H}, \mathrm{CH}_{3}\right) .{ }^{13} \mathrm{C} \mathrm{NMR}$ $\left(100 \mathrm{MHz}, \mathrm{DMSO}-d_{6}\right) \delta: 164.0(\mathrm{C}=\mathrm{O}), 159.2(\mathrm{C}-9), 156.0(\mathrm{C}-$ 7), 154.7 (C), 153.4 (C-2), 148.9 (C), 145.3 (C-4), 133.1 (C), 105.1 (C), 105.0 (C-6), $91.9(\mathrm{C}-8), 56.7\left(\mathrm{CH}_{3}\right), 56.2\left(\mathrm{CH}_{3}\right)$. MS (ESI), $m / z(\%): 290.1(100)[\mathrm{M}+\mathrm{H}]^{+}$. Anal. calcd for $\mathrm{C}_{13} \mathrm{H}_{11} \mathrm{~N}_{3} \mathrm{O}_{5}$ : C 53.98, H 3.83, N 14.53. Found: $\mathrm{C} 53.92, \mathrm{H}$ 3.79, N 14.50.

4.12. 4,7,9-Trihydroxy[1]benzofuro[3,2-d]pyrimidine-6carboxamide (13). A mixture of 4-hydroxy-7,9-dimethoxy [1]benzofuro[3,2-d]pyrimidine-6-carboxamide (7) (50 mg, $0.17 \mathrm{mmol}$ ) in warm pyridine hydrochloride $(3 \mathrm{~g})$ was irradiated using microwave $(100 \mathrm{~W})$ heating at $200^{\circ} \mathrm{C}$ for $10 \mathrm{~min}$. After addition of water, the mixture was extracted with ethyl acetate $(100 \mathrm{~mL})$. The organic layers were washed with brine, dried over sodium sulfate, filtered, and evaporated to dryness. The solvent was evaporated in vacuo, and the residue was purified by silica gel column chromatography using dichloromethane/methanol (9/1) as an eluent system to give compound 13 as a yellow solid (19 mg, 43\%). $R_{f}=0.41 \quad$ (dichloromethane/methanol: $9 / 1$ ). $\mathrm{Mp}=298-299^{\circ} \mathrm{C}$ (methanol). IR $\left(\mathrm{cm}^{-1}\right): 3464,3072,2883$, $1604,1402,1269,1195,1120 .{ }^{1} \mathrm{H}$ NMR (400 MHz, DMSO$\left.\mathrm{d}_{6}\right) \delta: 14.03(\mathrm{~s}, 1 \mathrm{H}, \mathrm{OH}), 12.89(\mathrm{~s}, 1 \mathrm{H}, \mathrm{OH}), 11.50(\mathrm{~s}, 1 \mathrm{H}$, $\mathrm{OH}), 8.40\left(\mathrm{~s}, 1 \mathrm{H}, \mathrm{CONH}_{2}\right), 8.21(\mathrm{~s}, 1 \mathrm{H}, \mathrm{H}-2), 7.44(\mathrm{~s}, 1 \mathrm{H}$, $\left.\mathrm{CONH}_{2}\right), 6.36$ (s, 1H, H-8). ${ }^{13} \mathrm{C}$ NMR $\left(100 \mathrm{MHz}, \mathrm{DMSO}-\mathrm{d}_{6}\right)$ $\delta: 169.6(\mathrm{C}=\mathrm{O}), 166.2(\mathrm{C}-4), 158.2(\mathrm{C}-7), 156.1(\mathrm{C}-9), 151.4$ (C), 146.9 (C-2), 144.0 (C), 136.8 (C), 104.0 (C), 99.1 (C-8), 92.9 (C). MS (ESI), $m / z(\%): 262.0(100)[\mathrm{M}+\mathrm{H}]^{+}$. Anal. calcd for $\mathrm{C}_{11} \mathrm{H}_{7} \mathrm{~N}_{3} \mathrm{O}_{5}$ : C 50.58, $\mathrm{H}$ 2.70, N 16.09. Found: C 50.42, H 2.71, N 16.13.

\section{Data Availability}

The data used to support the findings of this study are included within the article and in Supplementary Materials.

\section{Conflicts of Interest}

The authors declare that they have no conflicts of interest.

\section{Acknowledgments}

This research was supported by Vietnam National Foundation for Science and Technology Development (NAFOSTED) (10/2020/STS 01). The authors are thankful to T. Robiou du Pont for performing fungal seedings and cultures and M.C. Boumard for helping in separation and purification of cercosporamide. They also acknowledge Dr. B. Misme-Aucouturier for helping in statistical analysis.

\section{Supplementary Materials}

S1: ${ }^{1} \mathrm{H}$ NMR of 2,6-diiodo-3,5-dimethoxyphenol (2). S2: ${ }^{13} \mathrm{C}$ NMR of 2,6-diiodo-3,5-dimethoxyphenol (2). S3: ${ }^{1} \mathrm{H}$ NMR of ethyl (2,6-diiodo-3,5-dimethoxyphenoxy) acetate (3). S4: ${ }^{13} \mathrm{C}$ NMR of ethyl (2,6-diiodo-3,5-dimethoxyphenoxy) acetate (3). S5: ${ }^{1} \mathrm{H}$ NMR of ethyl (2,6-dicyano-3,5-dimethoxyphenoxy) acetate (4). S6: ${ }^{13} \mathrm{C}$ NMR of ethyl (2,6-dicyano-3,5dimethoxyphenoxy) acetate (4). S7: ${ }^{1} \mathrm{H}$ NMR of ethyl 
3-amino-7-cyano-4,6-dimethoxy-1-benzofuran-2-carboxylate (5). S8: ${ }^{13} \mathrm{NMR}$ of ethyl 3-amino-7-cyano-4,6-dimethoxy1-benzofuran-2-carboxylate (5). S9: ${ }^{1} \mathrm{H}$ NMR of 4hydroxy-7,9-dimethoxy[1]benzofuro[3,2-d]pyrimidine-6carbonitrile (6). S10: ${ }^{13} \mathrm{C}$ NMR of 4-hydroxy-7,9-dimethoxy [1]benzofuro[3,2-d]pyrimidine-6-carbonitrile (6). S11: ${ }^{1} \mathrm{H}$ NMR of 2-hydroxy-4,6-dimethoxybenzamide (8). S12: ${ }^{13} \mathrm{C}$ NMR of 2-hydroxy-4,6-dimethoxybenzamide (8). S13: ${ }^{1} \mathrm{H}$ NMR of 2-hydroxy-3-iodo-4,6-dimethoxybenzamide (9). S14: ${ }^{13} \mathrm{C}$ NMR of 2-hydroxy-3-iodo-4,6-dimethoxybenzamide (9). S15: ${ }^{1} \mathrm{H}$ NMR of 3-cyano-2-hydroxy-4,6dimethoxybenzamide (10). S16: ${ }^{13} \mathrm{C}$ NMR of 3-cyano2-hydroxy-4,6-dimethoxybenzamide (10). S17: ${ }^{1} \mathrm{H}$ NMR of ethyl (2-carbamoyl-6-cyano-3,5-dimethoxyphenoxy) acetate (11). S18: ${ }^{13} \mathrm{C}$ NMR of ethyl (2-carbamoyl-6-cyano-3,5dimethoxyphenoxy) acetate (11). S19: ${ }^{1} \mathrm{H}$ NMR of ethyl 3-amino-7-carbamoyl-4,6-dimethoxy-1-benzofuran-2-carboxylate (12). S20: ${ }^{13} \mathrm{C}$ NMR of ethyl 3-amino-7-carbamoyl4,6-dimethoxy-1-benzofuran-2-carboxylate (12). S21: ${ }^{1} \mathrm{H}$ NMR of 4-hydroxy-7,9-dimethoxy[1]benzofuro[3,2-d]pyrimidine-6-carboxamide (7). S22: ${ }^{13} \mathrm{C}$ NMR of 4 -hydroxy7,9-dimethoxy[1]benzofuro[3,2-d]pyrimidine-6-carboxamide (7). S23: ${ }^{1} \mathrm{H}$ NMR of 4,7,9-trihydroxy[1]benzofuro[3,2-d] pyrimidine-6-carboxamide (13). S24: ${ }^{13} \mathrm{C}$ NMR of 4,7,9trihydroxy[1]benzofuro[3,2-d]pyrimidine-6-carboxamide (13).

. (Supplementary Materials)

\section{References}

[1] M. A. Pfaller and D. J. Diekema, "Epidemiology of invasive candidiasis: a persistent public health problem," Clinical Microbiology Reviews, vol. 20, no. 1, pp. 133-163, 2007.

[2] A. A. Cleveland, L. H. Harrison, M. M. Farley et al., "Declining incidence of candidemia and the shifting epidemiology of Candida resistance in two US metropolitan areas, 2008-2013: results from population-based surveillance," PLoS One, vol. 10, no. 3, p. e0120452, 2015.

[3] M. Méan, O. Marchetti, and T. Calandra, "Bench-to-bedside review: Candida infections in the intensive care unit," Critical Care, vol. 12, no. 1, p. 204, 2008.

[4] M. Dadar, R. Tiwari, K. Karthik, S. Chakraborty, Y. Shahali, and K. Dhama, "Candida albicans-biology, molecular characterization, pathogenicity, and advances in diagnosis and control-an update," Microbial Pathogenesis, vol. 117, pp. 128-138, 2018.

[5] G. Sadeghi, M. Ebrahimi-Rad, and S. F. Mousavi, "Emergence of non-Candida albicans species: epidemiology, phylogeny and fluconazole susceptibility profile," Journal of Medical Mycology, vol. 28, no. 1, pp. 51-58, 2018.

[6] S. L. LaFayette, C. Collins, A. K. Zaas et al., "PKC signaling regulates drug resistance of the fungal pathogen Candida albicans via circuitry comprised of mkc1, calcineurin, and hsp90," PLoS Pathogens, vol. 6, no. 8, p. e1001069, 2010.

[7] J. Xie, N. Grahl, T. Sless et al., "Signaling through Lrg1, Rho1 and Pkcl governs Candida albicans morphogenesis in response to diverse cues," PLoS Genetics, vol. 12, no. 10, p. e1006405, 2016.

[8] F. Sugawara, S. Strobel, G. Strobel et al., "The structure and biological activity of cercosporamide from Cercosporidium henningsii," The Journal of Organic Chemistry, vol. 56, no. 3, pp. 909-910, 1991.
[9] A. Sussman, K. Huss, L.-C. Chio et al., "Discovery of cercosporamide, a known antifungal natural product, as a selective pkc1 kinase inhibitor through high-throughput screening," Eukaryotic Cell.vol. 3, no. 4, pp. 932-943, 2004.

[10] M. A. Conover, R. Mierzwa, A. King et al., "Usnic acid amide, a phytotoxin and antifungal agent from Cercosporidium henningsii," Phytochemistry, vol. 31, no. 9, pp. 2999-3001, 1992.

[11] V. Hung Dao, I. Garnier, M.-A. Bazin et al., "Benzofuro[3,2-d] pyrimidines inspired from cercosporamide $\mathrm{CaPkcl}$ inhibitor: synthesis and evaluation of fluconazole susceptibility restoration," Bioorganic \& Medicinal Chemistry Letters, vol. 28, 2018.

[12] P. M. Tadross, C. D. Gilmore, P. Bugga, S. C. Virgil, and B. M. Stoltz, "Regioselective reactions of highly substituted arynes," Organic Letters, vol. 12, no. 6, pp. 1224-1227, 2010.

[13] A. Bretéché, P. Marchand, M.-R. Nourrisson, G. D. Nanteuil, and M. Duflos, "A convenient route to functionalized 3amino-6-bromofuro[3,2-b]pyridine-2-carboxamides," Tetrahedron, vol. 66, no. 25, pp. 4490-4494, 2010.

[14] A. R. Pereira, W. K. Strangman, F. Marion et al., "Synthesis of phosphatidylinositol 3-kinase (pi3k) inhibitory analogues of the sponge meroterpenoid liphagal," Journal of Medicinal Chemistry, vol. 53, no. 24, pp. 8523-8533, 2010.

[15] S. Sato, M. Tetsuhashi, K. Sekine et al., "Degradation-promoters of cellular inhibitor of apoptosis protein 1 based on bestatin and actinonin," Bioorganic \& Medicinal Chemistry, vol. 16, no. 8, pp. 4685-4698, 2008.

[16] M. Bollier, F. Klupsch, P. Six et al., "One- or two-step synthesis of C-8 and N-9 substituted purines," The Journal of Organic Chemistry, vol. 83, no. 1, pp. 422-430, 2018.

[17] S. W. Grant, T. F. Gallagher, M. A. Bobko, C. Duquenne, and J. M. Axten, "Rapid synthesis of diversely functionalized 3,4,7trisubstituted indoles," Tetrahedron Letters, vol. 52, no. 26, pp. 3376-3378, 2011.

[18] R. S. Varma and K. P. Naicker, "The Urea-hydrogen peroxide complex: solid-state oxidative protocols for hydroxylated aldehydes and ketones (dakin reaction), nitriles, sulfides, and nitrogen heterocycles," Organic Letters, vol. 1, no. 2, pp. 189-192, 1999.

[19] K. J. McClure, L. Huang, K. L. Arienti et al., "Novel nonbenzimidazole chk2 kinase inhibitors," Bioorganic \& Medicinal Chemistry Letters, vol. 16, no. 7, pp. 1924-1928, 2006.

[20] M.-A. Bazin, L. Bodero, C. Tomasoni, B. Rousseau, C. Roussakis, and P. Marchand, "Synthesis and antiproliferative activity of benzofuran-based analogs of cercosporamide against non-small cell lung cancer cell lines," European Journal of Medicinal Chemistry, vol. 69, pp. 823832, 2013.

[21] T. H. Jepsen, M. Larsen, M. Jørgensen, and M. B. Nielsen, "Synthesis of dibenzothiophene, dibenzofuran and carbazole donor-acceptor chromophores," Synthesis, vol. 45, no. 8, pp. 1115-1120, 2013.

[22] S. Marhadour, P. Marchand, M. Antoine-Bazin et al., "Synthesis and biological evaluation of 2,3-diarylimidazo[1,2-a] pyridines as antileishmanial agents," European Journal of Medicinal Chemistry, vol. 58, pp. 543-556, 2012.

[23] A. M. Hoffman, S. G. Mayer, G. A. Strobel et al., "Purification, identification and activity of phomodione, a furandione from an endophytic Phoma species," Phytochemistry, vol. 69, no. 4, pp. 1049-1056, 2008. 